\title{
TNF- $\alpha$ downregulates eNOS expression and mitochondrial biogenesis in fat and muscle of obese rodents
}

\author{
Alessandra Valerio, ${ }^{1}$ Annalisa Cardile, ${ }^{1,2}$ Valeria Cozzi, ${ }^{1,2}$ Renata Bracale,1,2,3 Laura Tedesco, ,1,2,4 \\ Addolorata Pisconti, ${ }^{2,5}$ Letizia Palomba, ${ }^{6}$ Orazio Cantoni, ${ }^{6}$ Emilio Clementi, ${ }^{2,5,7}$ \\ Salvador Moncada, ${ }^{8}$ Michele O. Carruba, ${ }^{1,4}$ and Enzo Nisoli1,4 \\ 1Integrated Laboratories Network, Center for Study and Research on Obesity, Department of Pharmacology, School of Medicine, University of Milan, Milan, Italy. \\ ${ }^{2}$ Department of Preclinical Sciences, University of Milan, Milan, Italy. ${ }^{3}$ CEINGE Biotecnologie Avanzate, Naples, Italy. ${ }^{4}$ Istituto Auxologico Italiano, Milan, Italy. \\ ${ }^{5}$ Stem Cell Research Institute, San Raffaele Scientific Institute, Milan, Italy. ${ }^{6}$ Istituto di Farmacologia e Farmacognosia, University of Urbino “Carlo Bo," Urbino, \\ Italy. ${ }^{7}$ Eugenio Medea Scientific Institute, Lecco, Italy. ${ }^{8}$ Wolfson Institute for Biomedical Research, University College London, London, United Kingdom.
}

\begin{abstract}
Obesity is associated with chronic low-grade inflammation. Thus, at metabolically relevant sites, including adipose tissue and muscle, there is abnormal production of proinflammatory cytokines such as TNF- $\alpha$. Here we demonstrate that eNOS expression was reduced, with a concomitant reduction of mitochondrial biogenesis and function, in white and brown adipose tissue and in the soleus muscle of 3 different animal models of obesity. The genetic deletion of TNF receptor 1 in obese mice restored eNOS expression and mitochondrial biogenesis in fat and muscle; this was associated with less body weight gain than in obese wild-type controls. Furthermore, TNF- $\alpha$ downregulated eNOS expression and mitochondrial biogenesis in cultured white and brown adipocytes and muscle satellite cells of mice. The NO donors DETA-NO and SNAP prevented the reduction of mitochondrial biogenesis observed with TNF- $\alpha$. Our findings demonstrate that TNF- $\alpha$ impairs mitochondrial biogenesis and function in different tissues of obese rodents by downregulating eNOS expression and suggest a novel pathophysiological process that sustains obesity.
\end{abstract}

\section{Introduction}

Obesity is a complex, chronic disorder that has become a global epidemic (1). It is not just a concern for adults, as the number of overweight and obese children and adolescents has doubled in the past 2-3 decades in the United States. Visceral obesity, with a fat accumulation in abdomen among viscera, is often associated with insulin resistance, dyslipidemia, and hypertension and with an increased risk of accelerated atherosclerosis, i.e., the metabolic syndrome (1). It results from a positive energy balance in which more calories are consumed than are used up for oxidation (i.e., energy expenditure in skeletal muscle and brown adipose tissue [BAT]) or body building and maintenance, with consequent energy storage as fat (i.e., triglyceride accumulation in white adipose tissue [WAT]).

Obesity is associated with a state of chronic inflammation characterized by macrophage infiltration of muscle and adipose tissue (2) and abnormal production of proinflammatory mediators, including TNF- $\alpha(3,4)$ and iNOS (5). This inflammatory state is associated with a deficit of energy in the form of ATP $(6,7)$ and simultaneous overproduction of fat and leptin, which is accompanied by leptin resistance in the brain $(6,8)$.

We have previously shown that NO increases mitochondrial biogenesis, oxidative metabolism, and ATP levels in several cell

Nonstandard abbreviations used: BAT, brown adipose tissue; COX IV, cytochrome c oxidase IV; Ct, threshold cycle; Cyt c, cytochrome c; DETA-NO, (Z)-1-[2-(2-aminoethyl)-N-(2-ammonioethyl)amino]diazen-1-ium-1,2-diolate; DIO mice, high-fat diet-induced obese mice; mtDNA, mitochondrial DNA; NRF-1, nuclear respiratory factor-1; PGC-1 $\alpha$, peroxisome proliferator-activated receptor $\gamma$ coactivator $1 \alpha$; RNase, ribonuclease; SNAP, S-nitroso-acetyl penicillamine; Tfam, mitochondrial transcription factor A; WAT, white adipose tissue.

Conflict of interest: The authors have declared that no conflict of interest exists. Citation for this article: J. Clin. Invest. 116:2791-2798 (2006). doi:10.1172/JCI28570. types $(9,10)$. Consistently, mitochondrial biogenesis and function are markedly decreased in eNOS-null mutant ( $\mathrm{eNOS}^{-/-}$) mice $(9,10)$, with fat accumulation in the abdomen and body weight increased compared with wild-type animals (9). Here we investigated eNOS expression and mitochondrial biogenesis together with the role of TNF- $\alpha$ in different models of obesity. We demonstrate that both eNOS expression and mitochondrial biogenesis are downregulated in fat and muscle tissues of genetically and environmentally obese mice and rats. Moreover, we observed that the genetic deletion of TNF receptor 1 in obese mice restored eNOS expression and mitochondrial biogenesis in the same tissues. Thus, our findings indicate that TNF- $\alpha$ impairs mitochondrial biogenesis and function in metabolically active tissues of obese animals by inhibiting eNOS expression.

\section{Results}

eNOS expression and mitochondrial biogenesis in obese animals. eNOS mRNA and protein levels were measured in WAT from 3 animal models of obesity ( $o b / o b$ mice, $f a / f a$ rats, and high-fat diet-induced obese mice [DIO mice]) and were found to be significantly lower than those in the relevant controls (Figure 1). eNOS mRNA and protein levels were also significantly reduced in BAT and soleus muscle from $o b / o b$ mice, $f a / f a$ rats, and DIO mice, although the reduction was less than that in WAT (Supplemental Figure 1; supplemental material available online with this article; doi:10.1172/ JCI28570DS1). We next investigated whether eNOS down-regulation was accompanied by reduced mitochondrial biogenesis. Levels of mitochondrial DNA (mtDNA, a marker of mitochondrial content), peroxisome proliferator-activated receptor $\gamma$ coactivator $1 \alpha$ (PGC-1 $\alpha$ ), nuclear respiratory factor- 1 (NRF-1), and mitochondrial transcription factor A (Tfam; all master regulators of 
A

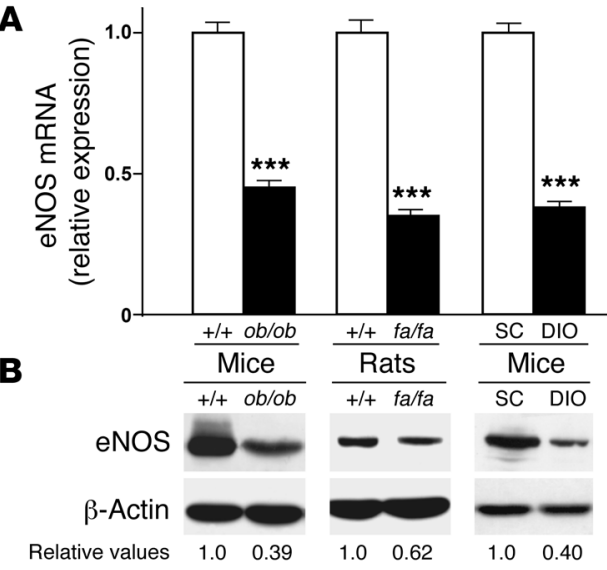

Figure 1

eNOS expression is reduced in WAT from obese animals. (A) eNOS mRNA levels, measured by means of quantitative RT-PCR in WAT of genetically obese mice (ob/ob) and rats ( $\mathrm{fa} / \mathrm{fa}$ ) and of environmentally obese mice (DIO) compared to respective controls (+/+, wild-type; SC, standard chow-fed animals). The cycle number at which the various transcripts were detectable was compared with that of $\beta$-actin as an internal control and expressed as arbitrary units versus values in control animals taken as 1.0 ( $n=5$ experiments). ${ }^{* * *} P<0.01$. (B) eNOS protein was detected by immunoblot analysis (1 experiment representative of 5 reproducible ones) in WAT of ob/ob mice, fa/fa rats, and DIO mice compared to respective controls. Numbers indicate the relative values from the densitometric analysis (normalized to $\beta$-actin) relative to controls, which were assigned a value of 1.0 . mitochondrial biogenesis; ref. 11) mRNA were markedly reduced in WAT, BAT, and soleus muscle of $o b / o b$ mice, $f a / f a$ rats, and DIO mice compared with controls (Figure 2A and Supplemental Figure 2, A-C). Similarly, the protein levels of cytochrome c oxidase IV (COX IV) and cytochrome c (Cyt c), 2 mitochondrial proteins involved in cell respiration, were significantly lower in obese than in lean control mice and rats, as was the oxygen consumption in most of the tissues studied (Figure 2, B and C, Supplemental Figure 2, D-F, and Supplemental Figure 3). These results are consistent with several prior reports showing that mitochondrial oxidative metabolism and mitochondrial gene expression (in particular PGC-1 $\alpha$ gene expression) are decreased in different tissues from both obese animals and humans (12).

We next investigated whether the decrease in respiration was associated with a decrease in ATP synthesis. The ATP levels of WAT, BAT, and soleus muscle of $o b / o b$ mice and $f a / f a$ rats were markedly lower than in controls (Table 1). Interestingly, although oxygen consumption of BAT from DIO

\section{Figure 2}

Mitochondrial biogenesis is reduced in WAT from obese animals. (A) PGC-1 $\alpha$, NRF-1, and Tfam mRNA, analyzed by means of quantitative RT-PCR with genespecific oligonucleotide probes in WAT of obese and control animals. The cycle number at which the various transcripts were detectable was compared to that of $\beta$-actin as an internal control, and expressed as arbitrary units versus values in control animals taken as 1.0 ( $n=5$ experiments). Shown at top is mtDNA analysis; 1 experiment representative of 5 gels $(n=5$ per group). Numbers indicate relative amounts from the densitometric analysis when control measurements were assigned a value of 1.0. Leftmost lanes show DNA markers (-). (B) COX IV and Cyt c proteins were detected by immunoblot analysis (1 experiment representative of 5 reproducible ones) in WAT of obese and control animals. Numbers indicate relative values from the densitometric analysis (normalized to $\beta$-actin) relative to controls, which were assigned a value of 1.0. (C) Oxygen consumption by WAT of obese and control animals was measured in a gas-tight chamber using an $\mathrm{O}_{2}$ electrode. Oxygen consumption values were normalized to the tissue protein content $(n=3$ experiments). ${ }^{\star *} P<0.01,{ }^{*} P<0.05$.

B

C
A

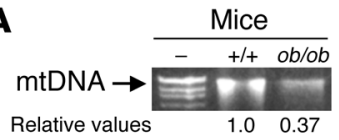

mice was not significantly different from that of controls, ATP levels were significantly reduced (Table 1 ), confirming previously reported results (13) and suggesting that the high lipid content of the diet can directly affect BAT respiration $(14,15)$. Finally, we measured the mitochondrial mass in $o b / o b$ and lean control mice by morphometric analysis of mitochondria at the ultrastructural level. In WAT (Figure 3) as well as in BAT and soleus muscle (Supplemental Figure 4), the mitochondrial area was markedly reduced in $o b / o b$ mice compared with that of lean controls. Mitochondrial density was reduced in WAT (Figure 3), but not in BAT (data not shown), and was slightly increased in soleus muscle (Supplemental Figure 4). Furthermore, mitochondrial cristae length was strongly
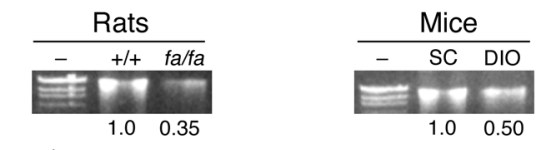
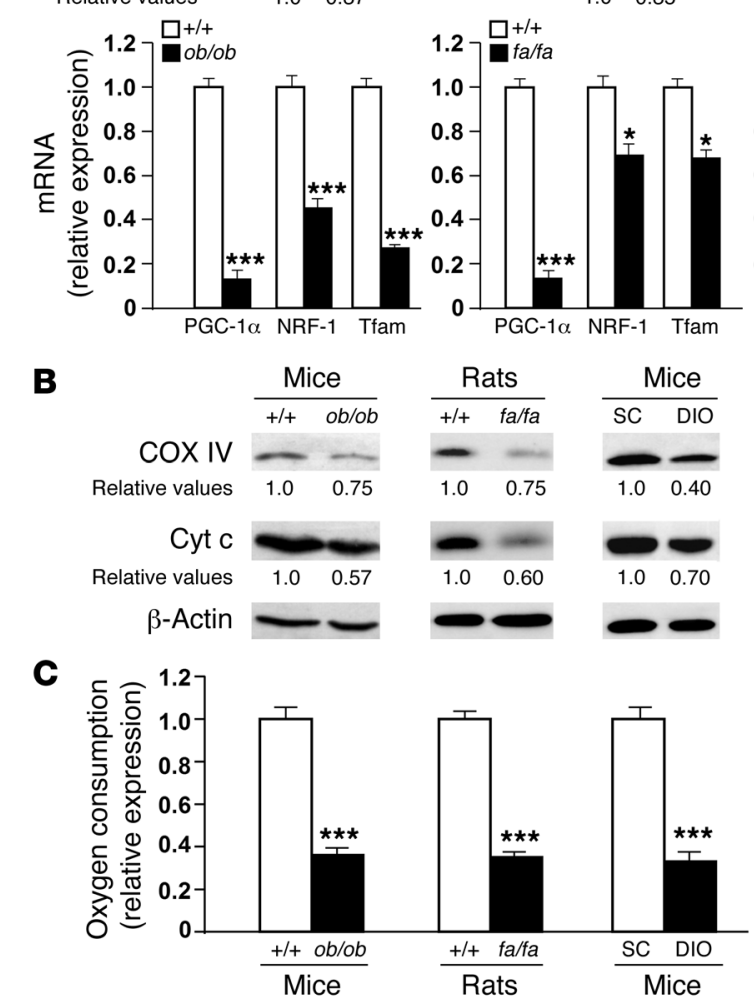


\section{Table 1}

ATP levels in tissues from both obese and control rodents

\begin{tabular}{|c|c|c|c|c|c|c|}
\hline \multirow[t]{2}{*}{ Tissue } & \multicolumn{6}{|c|}{ ATP (nmol/mg tissue) } \\
\hline & Wild-type mice & ob/ob mice & Wild-type rats & fa/fa rats & SC mice & DIO mice \\
\hline WAT & $0.051 \pm 0.005$ & $0.025 \pm 0.008^{A}$ & $0.058 \pm 0.004$ & $0.019 \pm 0.004^{A}$ & $0.051 \pm 0.006$ & $0.023 \pm 0.005^{A}$ \\
\hline BAT & $0.298 \pm 0.002$ & $0.147 \pm 0.010^{\mathrm{A}}$ & $0.330 \pm 0.005$ & $0.161 \pm 0.008^{\mathrm{B}}$ & $0.213 \pm 0.030$ & $0.177 \pm 0.080^{B}$ \\
\hline Soleus muscle & $4.530 \pm 0.910$ & $3.720 \pm 0.710^{B}$ & $4.210 \pm 0.180$ & $3.390 \pm 0.080^{B}$ & $4.670 \pm 0.520$ & $3.310 \pm 0.120^{\mathrm{B}}$ \\
\hline
\end{tabular}

Steady-state levels of ATP were measured as described in Methods. SC mice, standard chow-fed mice. $n=3-4$ per group. ${ }^{A} P<0.01$ versus respective control. $\mathrm{B} P<0.05$ versus respective control.

reduced in BAT (particularly in unilocular cells), and smaller differences were also evident in WAT and soleus muscle (Figure 3 and Supplemental Figure 4). These findings indicate that mitochondrial biogenesis and function are significantly diminished in fat and muscle of obese animals.

eNOS expression and mitochondrial biogenesis in obese animals with TNF- $\alpha$ signaling deficiency. Since fat and muscle TNF- $\alpha$ production is increased in obesity $(3,4)$, we investigated both eNOS expression and mitochondrial biogenesis in WAT and BAT of obese mice in which the signaling and function of TNF- $\alpha$ were partially abolished, i.e., $o b / o b$ mice lacking either the p55 or the p75 TNF recep-

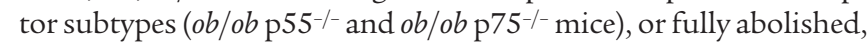

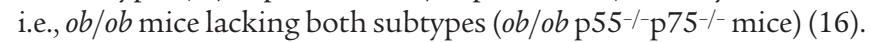
eNOS mRNA and protein levels in both WAT and BAT of the $o b / o b$ $\mathrm{p} 55^{-/-}$and $o b / o b \mathrm{p} 55^{-/-} \mathrm{p} 75^{-/-}$mice were significantly higher than in those of $o b / o b$ mice wild-type at TNF receptor loci and $o b / o b$ p $75^{-/-}$mice, and they exceeded the levels observed in the control lean mice (increases in mRNA and protein levels, respectively: $o b / o b$ p55-/- WAT, $34 \% \pm 4 \%$ and $24 \% \pm 6 \%$; ob/ob p55/- $75^{-/-}$WAT, $23 \% \pm 5 \%$ and $19 \% \pm 4 \%$; ob/ob p $55^{-/-}$BAT, $36 \% \pm 2 \%$ and $43 \% \pm 5 \%$; $o b / o b$ p $55^{-/-}$p $75^{-/-}$BAT, $33 \% \pm 5 \%$ and $29 \% \pm 3 \%$; Figure 4 A and Supplemental Figure 5A). A slight but statistically significant restoration of the mtDNA amounts and the PGC- $1 \alpha$, NRF-1, and Tfam mRNA levels was evident in WAT and BAT of both $o b / o b \mathrm{p} 55^{-/-}$and $o b / o b \mathrm{p} 55^{-/-} \mathrm{p} 75^{-/-}$mice, but not in WAT and BAT of $o b / o b \mathrm{p} 75^{-/-}$ mice (Figure 4B and Supplemental Figure 5B). Similarly, the protein levels of COX IV and Cyt c in both WAT and BAT of the $o b / o b$ $\mathrm{p} 55^{-/-}$and $o b / o b \mathrm{p} 55^{-/-} \mathrm{p} 75^{-/-}$mice were significantly higher than in those of $o b / o b$ mice wild-type at TNF receptor loci and $o b / o b \mathrm{p}^{75^{-/-}}$ mice (Figure 4C and Supplemental Figure 5C).

We developed an additional model of obesity in which TNF- $\alpha$ signaling was defective, i.e., p55/- mice fed a high-fat diet for 3 months (DIO p55/- mice). eNOS mRNA expression, oxygen consumption, and ATP production were measured in these obese mice and compared with those observed in age- and sex-matched DIO mice wild-type at TNF receptor loci as well as with mice fed a standard diet. All 3 parameters were significantly higher in WAT of those DIO p55/- mice that gained less body weight than

\section{Figure 3}

Mitochondrial pattern is altered in WAT of ob/ob mice. WAT from control or $o b / o b$ mice was processed to perform stereological analysis of mitochondria. The tissues were fixed and sectioned randomly and prepared for electron microscopy. Representative electron micrographs are shown at left. M, mitochondria; L, lipid droplets. At right, 50 photographs at a magnification of $\times 11,500$ were taken, and the area and density of 998 wild-type and 1,112 ob/ob mitochondria were determined. Results are mean $\pm \operatorname{SEM}\left(n=3\right.$ per group). ${ }^{* \star *} P<0.01,{ }^{*} P<0.05$. the wild-type DIO mice (Figure 5, A-C). Oxygen consumption and ATP production were also significantly higher in BAT and soleus muscle of the DIO p55/-- mice (Supplemental Figure 6, A and B). No statistically significant difference in these parameters was evident in those DIO p55/- mice that gained body weight at a rate similar to that of the wild-type DIO mice ( $~ 30 \%$ of DIO p55/- mice) (data not shown). These results demonstrate that the activity of TNF- $\alpha$ is a primary determinant of the WAT, BAT, and skeletal muscle downregulation of eNOS expression and impaired mitochondrial biogenesis in obesity, since defective TNF- $\alpha$ signaling confers protection from the obesity-induced decline in both eNOS and mitochondrial function. The p55 receptor appears to be involved in mediating the effects of TNF- $\alpha$.

Effects of TNF- $\alpha$ on eNOS expression and mitochondrial biogenesis in fat and muscle cells. A reduction in eNOS and mitochondrial biogenesis was also observed when TNF- $\alpha$ was administered to WAT and BAT cells and muscle satellite cells (the skeletal muscle progenitor cells responsible for postnatal growth and repair; ref. 17) differentiated in culture. eNOS mRNA and protein levels were both decreased in a concentration-dependent manner by exposure of the cells to TNF- $\alpha$ for 2 days (Figure 6A and Supplemental Figure 7A). This effect was particularly evident in WAT cells, in which the eNOS mRNA and protein levels were decreased by $66 \% \pm 2 \%$ and $48 \% \pm 3 \%$, respectively, after treatment with $10 \mathrm{nM}$ TNF- $\alpha$ (Figure 6A). The decrease in eNOS was paralleled by a concomitant decrease in mitochondrial biogenesis, as demonstrated by a significant reduction of PGC-1 $\alpha, \mathrm{NRF}-1$, and Tfam mRNA levels in WAT cells exposed for 2 days to $1 \mathrm{nM}$ TNF- $\alpha$ (Figure 6B). COX IV and Cyt $\mathrm{c}$ protein levels in WAT cells were also reduced significantly by $1 \mathrm{nM}$ TNF- $\alpha$ (Figure 6C). TNF- $\alpha$-induced reduction of COX IV and Cyt $\mathrm{c}$ was statistically significant in both BAT and satellite muscle cells, but in some cases was less marked than in WAT cells
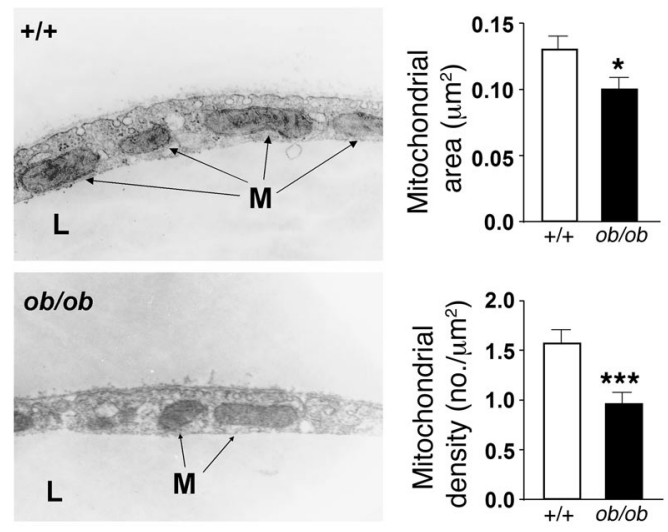

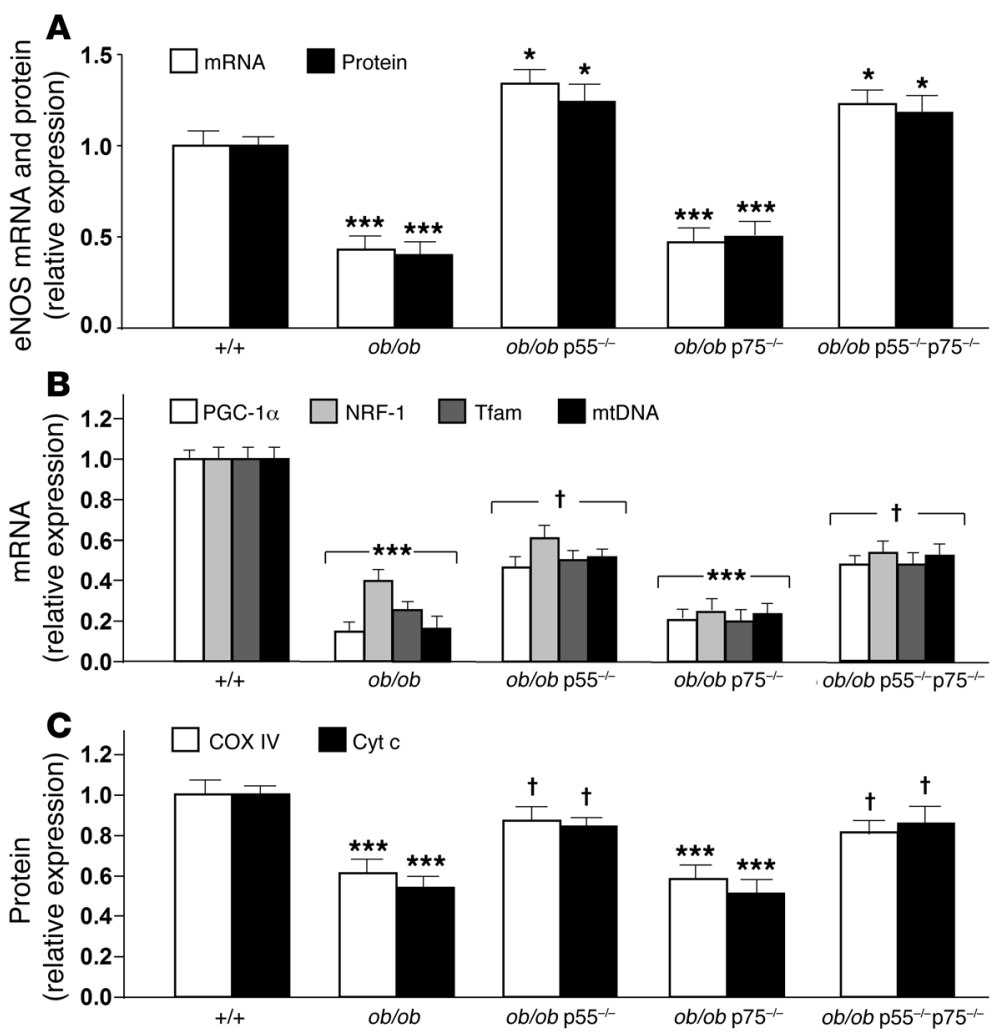

\section{Figure 4}

TNF- $\alpha$ signaling deficiency partly restores mitochondrial biogenesis in genetic obesity. (A) eNOS mRNA and protein levels were analyzed by quantitative RT-PCR or immunoblotting, respectively, in WAT of wild-type, ob/ob,

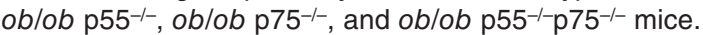
Relative values are mean \pm SEM ( $n=3$ per group) when control measurements were assigned a value of 1.0. (B) PGC-1 $\alpha, N R F-1$, and Tfam mRNA were analyzed by means of quantitative RT-PCR with gene-specific oligonucleotide probes in WAT of wild-type, ob/ob, ob/ob p55--1,

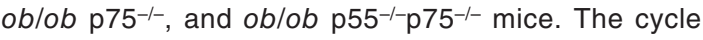
number at which the various transcripts were detectable was compared with that of $\beta$-actin as an internal control and expressed as arbitrary units. (C) COX IV and Cyt C protein levels were detected by densitometric analysis of immunoblots in WAT of wild-type, ob/ob, ob/ob p55-1-,

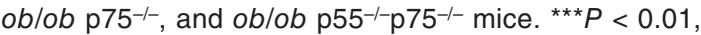
${ }^{*} P<0.05$ versus wild-type ( $n=3$ per group); ${ }^{\dagger} P<0.05$ versus ob/ob.

recently been suggested as a major cause $(6,7,19,20)$. Here we have shown that both eNOS expression and mitochondrial biogenesis were downregulated in metabolically active tissues, including WAT, BAT, and skeletal muscle, of both genetically and high-fat diet-fed obese rodents. The reduced mitochondrial function is compatible with the defective lipid oxidation seen in obese patients (21).

We have previously reported that eNOS-dependent

(Supplemental Figure 7B). The concentrations of TNF- $\alpha$ used in these experiments are comparable with the $15-48 \mathrm{nM}$ present in the plasma of lean and obese rodents (3).

To demonstrate further that TNF- $\alpha$ impairs mitochondrial biogenesis, we measured the oxygen consumption in WAT, BAT, and muscle satellite cells. Interestingly, a strong concentration-dependent decrease was evident in TNF- $\alpha$-treated WAT cells (Figure 6D) and muscle satellite cells (Supplemental Figure 7C). Fewer marked changes were observed in BAT cells, in which, furthermore, the highest TNF- $\alpha$ concentration $(10 \mathrm{nM})$ did not change the oxygen consumption compared with untreated cells (Supplemental Figure 7C). This might be due to specific actions of TNF- $\alpha$ in these cells, including modulation of uncoupling proteins that regulate respiration of BAT cells (18). These results strongly suggest that TNF- $\alpha$ may be involved in the mitochondrial biogenesis deficit we observed in different tissues of obese animals.

To verify that the downregulation of eNOS by TNF- $\alpha$ is a relevant molecular mechanism by which mitochondrial biogenesis is affected, fat and muscle cells were treated with TNF- $\alpha$ in the presence of 2 structurally unrelated NO donors. (Z)-1-[2-(2-aminoethyl)-N-(2-ammonioethyl)amino]diazen-1-ium-1,2-diolate (DETA-NO; $50 \mu \mathrm{M})$ prevented the effect of TNF- $\alpha$ on mitochondrial biogenesis, with full restoration of PGC-1 $\alpha$, NRF-1, Tfam, COX IV, and Cyt c expression as well as oxygen consumption in fat and muscle cells (Figure 6, B-D, Supplemental Figure 7, B and C, and data not shown). Similar results were obtained with S-nitrosoacetyl penicillamine (SNAP; $100 \mu \mathrm{M}$; data not shown).

\section{Discussion}

Obesity is a complex epidemic disorder with a poorly understood pathophysiology (1). Defective cellular energy metabolism has
NO production induces mitochondrial biogenesis, with a concomitant increase of PGC-1 $\alpha$, NRF-1, and Tfam gene expression, oxygen consumption, and ATP production in adipose and muscle cells (9, 10). Conversely, eNOS $-/-$ mice showed a marked deficit in mitochondrial biogenesis and developed visceral obesity $(9,10)$ and metabolic syndrome (22). Consistent with our present observations, a 3.7-fold decrease of eNOS expression in total abdominal WAT of male DIO mice (23) and an inverse correlation among the skeletal muscle eNOS content, percent body fat, and body mass index in young adult women (24) have previously been described. In this context, dietary supplementation with L-arginine, which increases serum NO concentration and PGC-1 $\alpha$ expression in WAT, has been shown to reduce fat mass in Zucker diabetic fatty rats 4-10 weeks after the initiation of treatment compared with control rats (25).

Confirming that mitochondrial dysfunction is relevant for the pathophysiology of obesity, large-scale gene-expression analyses performed in obese animals $(19,26,27)$ and humans $(28,29)$ have demonstrated that many genes encoding mitochondrial proteins are negatively correlated with body mass (30). Additionally, mice with genetic deletion of NEIL1 DNA glycosylate, an enzyme involved in base excision repair of ring-fragmented purines and some pyrimidines, have increased mtDNA damage and deletions and develop severe obesity with dyslipidemia, fatty liver disease, and a tendency toward hyperinsulinemia (31).

Moreover, obese patients are deficient in energy in the form of ATP $(6,32)$. Such low ATP levels are associated with increased appetite and decreased exercise capacity, with high fatigability (33). Recently, low and high aerobic treadmill-running capacity was selected in genetically heterogeneous rats (20), with low-capacity runners showing higher visceral adiposity, blood pressure, insulin resistance, plasma triglycerides, and free fatty 
A

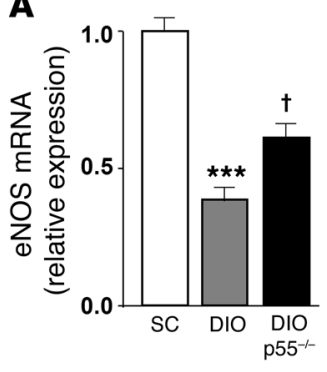

B

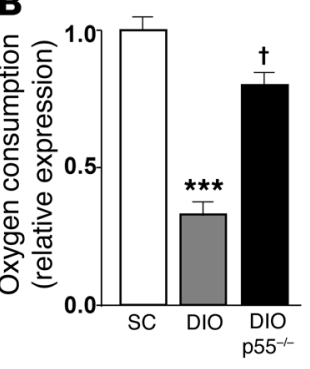

C

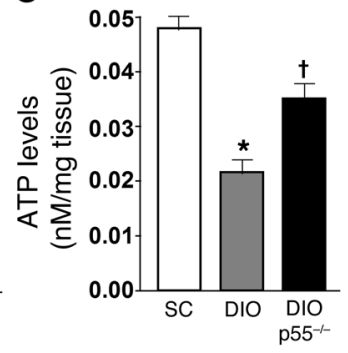

Figure 5

TNF- $\alpha$ signaling deficiency partly restores mitochondrial biogenesis in diet-induced obesity. (A) eNOS mRNA analyzed by quantitative RT-PCR in WAT of standard chow-fed mice (control), DIO mice, or DIO p55--- mice. (B) Oxygen consumption and (C) ATP levels in WAT of DIO and DIO p55 $5^{-/-}$mice were compared with those of standard chow-fed mice. Relative values are mean \pm SEM ( $n=3$ per group) when control measurements were assigned a value of 1.0 . ${ }^{* *} P<0.01$, ${ }^{*} P<0.05$ versus standard chow; ${ }^{\top} P<0.05$ versus $\mathrm{DIO}$. acids compared with those of the high-capacity runners. Intriguingly, mitochondrial dysfunction, defective oxidative metabolism, and low mitochondrial gene expression were evident in low-capacity runners (20), suggesting that visceral obesity and related disorders are linked to defective mitochondrial biogenesis and oxidative metabolism with decreased ATP production. Our findings support this hypothesis, not only at the molecular and biochemical levels but also at the morphological level. Mitochondria were typically large and round or oval with wellorganized cristae in WAT, BAT, and soleus muscle of control mice. In contrast, mitochondrial cristae were pleomorphic and disorganized in BAT of obese mice, although the typical mitochondrial shape was conserved in both WAT and BAT. In soleus muscle of $o b / o b$ mice, however, the mitochondria were small, heterogeneous, elongated, and bent, and the cristae were folded (Supplemental Figure 4). This morphology, which paralleled the functional deficits of mitochondria, suggested an increase of the fission events in mitochondrial dynamics. In this context, the expression of mitofusin 2, which is involved in the mitochondrial fusion events, has previously been reported to be decreased in the skeletal muscle of obese $f a / f a$ rats (34).

TNF- $\alpha$, which is overproduced in the adipose as well as muscle tissues of obese patients $(3,4,26,27)$, plays a significant role in these processes. In fact, mitochondrial biogenesis in WAT, BAT, and muscle was at least partially restored in the obese mice with defective TNF- $\alpha$ signaling. This partial recovery of mitochondrial biogenesis in WAT and BAT of both $o b / o b \mathrm{p} 55^{-/-}$and $o b / o b \mathrm{p} 55^{-/-} \mathrm{p} 75^{-/-}$mice was accompanied by full restoration of eNOS expression in these tissues. Conversely, only partial restoration

\section{Figure 6}

Mitochondrial biogenesis is downregulated by TNF- $\alpha$ and restored by an NO donor in WAT cells. (A) eNOS mRNA and protein were analyzed by quantitative RT-PCR and immunoblotting, respectively, in untreated cells $(C$, control) or after exposure to TNF- $\alpha$ at different doses for 2 days. (B) PGC-1 $\alpha$, NRF-1, and Tfam mRNA levels were determined in untreated WAT cells and after treatment with $1 \mathrm{nM}$ TNF- $\alpha$ alone or in combination with $50 \mu \mathrm{M}$ DETA-NO for 2 days. (C) COX IV and Cyt c protein levels and (D) oxygen consumption were measured in untreated WAT cells and after treatment with $1 \mathrm{nM}(\mathbf{C})$ or different doses (D) of TNF- $\alpha$ alone or in combination with $50 \mu \mathrm{M}$ DETA-NO for 2 days. Relative values are mean \pm SEM ( $n=4$ different experiments) when control measurements were assigned a value of 1.0. ${ }^{\star \star} P<0.01,{ }^{\star} P<0.05$ versus untreated cells. of eNOS expression accompanied the partial recovery of mitochondrial biogenesis in WAT of DIO $\mathrm{p}^{55^{-/}}$mice, possibly reflecting pathophysiological differences between $o b / o b$ and DIO mice. Moreover, TNF- $\alpha$ markedly decreased both eNOS expression and mitochondrial biogenesis in cultured fat and muscle cells. Notably, the downregulation of eNOS is the major molecular mechanism by which TNF- $\alpha$ affects mitochondrial biogenesis in in vitro models, as clearly shown by the full reversal of the effects of TNF- $\alpha$ on mitochondria by the NO donors DETA-NO and SNAP. Together, these results suggest that TNF- $\alpha$, even if not the sole factor involved, plays a relevant role in decreasing eNOS expression and mitochondrial biogenesis in metabolically active

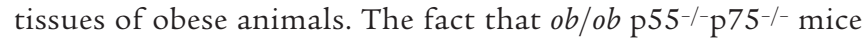

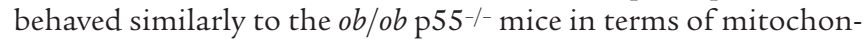
drial parameters suggests that p55 is the most relevant receptor in mediating the TNF- $\alpha$ effects.

Large-scale gene-expression analyses and immunohistochemistry have demonstrated that macrophages are accumulated in patches in the expanding adipose tissue $(26,27,35)$, with an increased release of inflammatory mediators, including TNF- $\alpha$ and iNOS $(26,27)$. It has been observed that upregulation of iNOS (which is induced in inflammatory conditions) often cor-
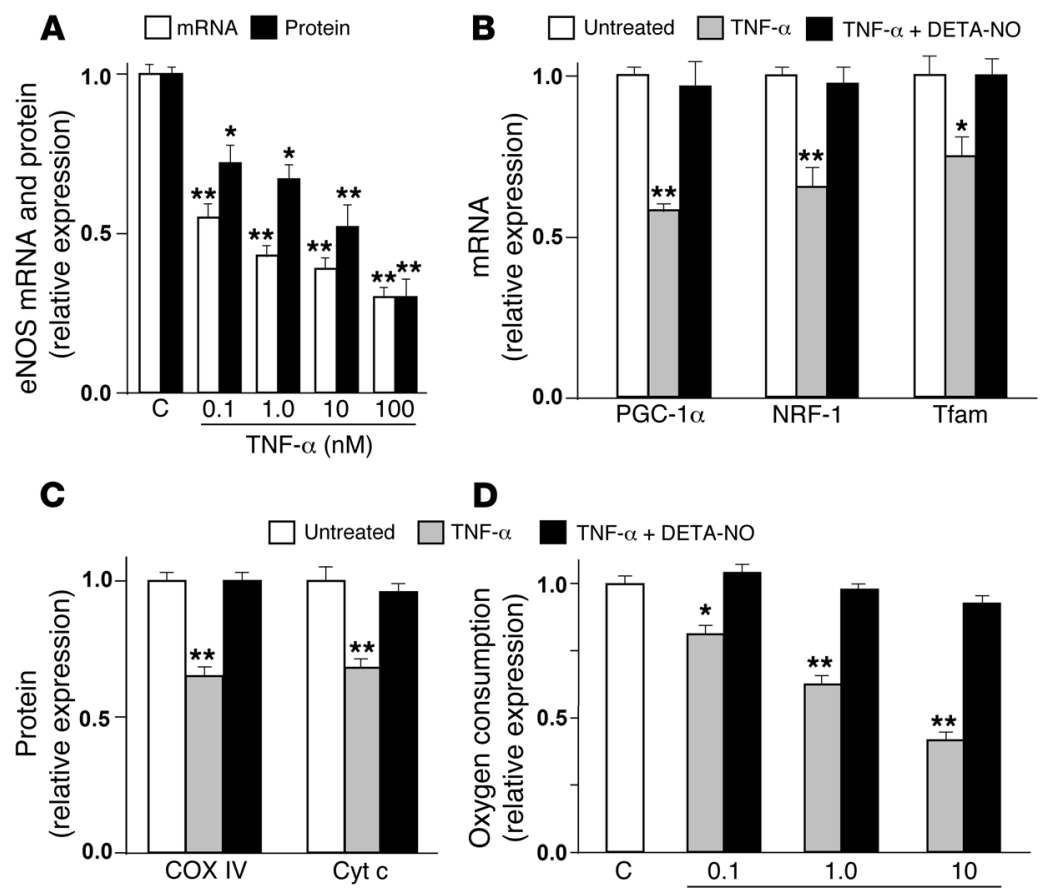

D

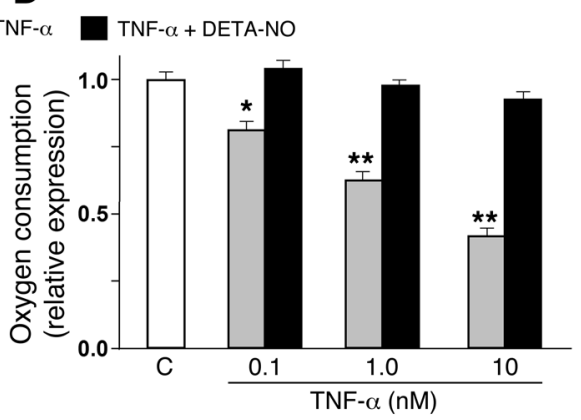


relates with downregulation of eNOS (36). Corroborating this, TNF- $\alpha$ increases iNOS expression in different cells and tissues, including fat and muscle (37), and inhibits eNOS expression, mitochondrial function, and energy production not only in WAT, BAT, and muscle cells, but also in endothelial cells, cardiac myocytes, and fibroblast cell lines (38-40). Recently it has been demonstrated that TNF- $\alpha$ can positively autoregulate its own biosynthesis in adipose tissue, contributing to the maintenance of elevated TNF- $\alpha$ in obesity (41). Interestingly, many obese patients show elevated levels of C-reactive protein as a result of bacterial or fungal infection (42) in addition to increased TNF- $\alpha$ production. Moreover, some toxins - which are widespread in the environments of ind ustrialized countries - at environmental concentrations increase TNF- $\alpha$ levels in the serum and livers of exposed rhesus monkeys or in different cells in culture, including adipocytes, monocytes, and macrophages (6). These results suggest that environmental agents (infections and toxins) can also play a role in the pathophysiology of obesity.

Overall these findings suggest that the inadequate body energy disposal (refs. 7, 43, and our present results) and the impaired anorexigenic effect of adipose leptin due to leptin resistance (8) convey to the brains of obese patients an "emergency" signal difficult to ignore (44), namely, that more energy is needed to survive. As a consequence, obese patients increase their food intake and restrict physical activity in order to maintain energy supply to the body (6). Thus, although overeating and a sedentary lifestyle lead to fat accumulation, they might not be the only pathophysiological bases of obesity. Conversely, they may be the result of an already "obese metabolism," which would force individuals to overeat and preserve energy (6). This hypothesis may be supported by the observation that while in normal-weight rats and mice moderate calorie restriction enhances mitochondrial oxidative capacity (45) by increasing eNOS expression and mitochondrial biogenesis (46), in obese patients a restrictive diet further lowers the rate of already defective lipid oxidation in different tissues (47), probably due to the lack of the compensatory NO-related mitochondrial biogenesis (our present results). Our findings therefore suggest the need for an alternative therapeutic approach to be investigated in obese animals and humans, including that of increasing mitochondrial mass and function.

\section{Methods}

Animals. Male 8-week-old mice of the genetically obese model $o b / o b$ in the C57BL/6J strain, along with littermate controls, were purchased from Harlan. These mice were fed standard chow. For DIO studies, starting at 4-5 weeks of age, wild-type C57BL/6J mice from Harlan were put on diets containing $60 \% \mathrm{kcal}$ from fat (The Jackson Laboratory) for 3 months. Male and female 4- to 5-month-old obese $(o b / o b)$ mice and $o b / o b$

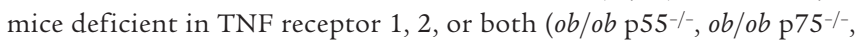
and $o b / o b \mathrm{p} 55^{-/} \mathrm{p} 75^{-/-}$mice) were a generous gift from G.S. Hotamisligil (Harvard School of Public Health, Boston, Massachusetts, USA) (16). Male 8-week-old TNF receptor 1-null mutant mice (p55-/-; The Jackson Laboratory) were put on a diet containing 60\% kcal from fat for 3 months (DIO p55/- mice). Male 5-week-old Zucker-fa/fa/Ola obese rats and Zucker-+/+/Ola controls were obtained from Charles River Laboratories. Animals were treated according to protocols approved by Milan University Institutional Animal Care and Use Committee. On the day of the experiments, animals were sacrificed by either cervical dislocation (mice) or decapitation (rats), and tissues were immediately isolated. They were either frozen in liquid nitrogen (for mtDNA, mRNA, and ATP studies); fixed (for electron microscopy); or freed of connective tissue and large vasculature, cut into $30-20 \mathrm{mg}$ slices and maintained in a Krebs-Ringer solution (containing $118 \mathrm{mM} \mathrm{NaCl} ; 4.7 \mathrm{mM} \mathrm{KCl} ; 1.5 \mathrm{mM} \mathrm{CaCl}_{2} ; 25 \mathrm{mM}$ $\mathrm{NaHCO}_{3} ; 1.2 \mathrm{mM} \mathrm{KH}_{2} \mathrm{PO}_{4} ; 1.1 \mathrm{mM} \mathrm{MgSO}_{4}$; and $10 \mathrm{mM}$ glucose, $\mathrm{pH} 7.4$ ) in which $20 \% \mathrm{O}_{2}, 5 \% \mathrm{CO}_{2}$, and $75 \% \mathrm{~N}_{2}$ was continuously bubbled at $37^{\circ} \mathrm{C}$ (for $\mathrm{O}_{2}$ consumption experiments).

WAT and BAT cell and muscle satellite cell isolation, culture, and treatment. BAT and WAT precursor cells were enzymatically isolated from the interscapular BAT and epididymal WAT of wild-type C57BL/6J mice, which were kept in standard laboratory conditions (12-hour light/dark cycle; food and water ad libitum), as previously described $(9,48)$. Mouse satellite cells were prepared from limbs of 1- to 2-week-old mice as previously described (49). The cells were grown in DMEM supplemented with $20 \%$ horse serum (HS) and 5\% chicken embryo extract (EE). To induce differentiation, the cells were shifted to DMEM supplemented with $5 \%$ HS and $1.25 \% \mathrm{EE}$ for $3-5$ days. In the experiments analyzing the effects of TNF- $\alpha$, the cultured cells were exposed to the culture medium containing either murine recombinant TNF- $\alpha$ (freshly diluted in buffer as instructed by the manufacturer; Genzyme) or TNF- $\alpha$ at different concentrations plus $50 \mu \mathrm{M}$ DETA-NO (or $100 \mu \mathrm{M}$ SNAP) for 2 days and then harvested. The medium was discarded, the wells were washed twice with $2 \mathrm{ml}$ ice-cold PBS (Sigma-Aldrich), and the cells were scraped out with a disposable cell scraper for mRNA extraction and RT-PCR analysis.

Quantitative RT-PCR. Analysis of mRNA levels was performed as described previously (46). One microgram of total RNA, isolated from the different tissues using the RNeasy Lipid (or Fibrous) Tissue Mini Kit (QIAGEN), was treated for 1 hour at $37^{\circ} \mathrm{C}$ with $6 \mathrm{U}$ ribonuclease-free (RNase-free) deoxyribonuclease I per $\mu \mathrm{g}$ RNA in $100 \mathrm{mM}$ Tris- $\mathrm{HCl}, \mathrm{pH} 7.5$, and $50 \mathrm{mM}$ $\mathrm{MgCl}_{2}$ in the presence of $2 \mathrm{U} / \mu \mathrm{l}$ placental RNase inhibitor; it was then reverse transcribed using iScript cDNA Synthesis Kit (Bio-Rad). Primers were designed using Beacon Designer 2.6 software from Premier Biosoft International. Triplicate PCR reactions were carried out with the intercalating dye SYBR Green. Each sample $(25 \mu \mathrm{l})$ contained $12.5 \mu \mathrm{l}$ iQ SYBR Green I Supermix (Bio-Rad), $0.4 \mu \mathrm{M}$ each primer, and one-fiftieth reverse transcriptase product. PCR cycles were programmed on an iCycler iQ Real Time PCR Detection System (Bio-Rad). The cycle number at which the various transcripts were detectable, referred to as the threshold cycle $(\mathrm{Ct})$, was compared with that of $\beta$-actin and referred to as $\Delta \mathrm{Ct}$. The gene relative level was expressed as $2^{-(\Delta \Delta \mathrm{Ct})}$, in which $\Delta \Delta \mathrm{Ct}$ equals $\Delta \mathrm{Ct}$ of the obese mice (or treated cells) minus $\Delta \mathrm{Ct}$ of the control mice (or untreated cells).

Immunoblot analysis. After extraction, equal amounts of protein were run on SDS-PAGE under reducing conditions. The separated proteins were then electrophoretically transferred to a nitrocellulose membrane (Protran; Schleicher \& Schuell). Proteins of interest were revealed with specific antibodies: anti-Cyt c (BD Biosciences), anti-COX IV (Invitrogen), antieNOS (BD Biosciences) at 1:500 dilution and anti- $\beta$-actin (Sigma-Aldrich) at 1:5,000 dilution. The immunostaining was detected using horseradish peroxidase-conjugated anti-rabbit immunoglobulin and anti-mouse immunoglobulin for 1 hour at room temperature. Bands were revealed by the SuperSignal Substrate (Pierce Biotechnology) and quantitated by densitometry using a Quick Image densitometer (Canberra Packard) and Phoretix 1D version 3.0 image analyzer software.

$m t D N A$ analysis. Mitochondria were isolated from tissues and cells. The mtDNA was extracted and analyzed as described $(9,46)$. Mitochondria were lysed in $10 \mathrm{mM}$ Tris- $\mathrm{HCl}$ (pH 8.0), $20 \mathrm{mM}$ EDTA, and $0.5 \%$ Triton X 100 $(\mathrm{pH} 8.0)$ and occasionally shaken while on ice for 30 minutes. The mtDNA was extracted with a mixture of phenol and chloroform, one-tenth volume of $5 \mathrm{mM} \mathrm{NaCl}$ was added to the solution, and $\mathrm{mtDNA}$ was then precipitated overnight by adding an equal volume of isopropanol. After overnight precipitation at $-20^{\circ} \mathrm{C}, \mathrm{mtDNA}$ was pelletted by centrifugation, resuspended in sterile water, and treated with $50 \mu \mathrm{g} / \mathrm{ml}$ RNase. An aliquot of mtDNA 
was loaded on ethidium bromide-stained agarose gel (1.2\%) and analyzed using the Quick Image densitometer (Canberra Packard). The mtDNA levels were determined from the signal intensity and the cell number from each treatment (or the protein content from each tissue obtained from wild-type and knockout mice). Each sample was also measured by UV absorption at $260 \mathrm{~nm}$ with a Jasco V520 spectrophotometer. mtDNA copy numbers were also measured by means of quantitative RT-PCR from the cytochrome $\mathrm{c}$ oxidase II mtDNA gene in WAT and BAT of $o b / o b$ p55/-, ob/ob $\mathrm{p}^{-/ /-}, o b / o b$ p55-/- p75-/, and control mice as described previously (19).

Measurement of oxygen consumption. We analyzed 1-ml cell or tissue samples at $37^{\circ} \mathrm{C}$ in a gas-tight vessel equipped with a Clark-type oxygen electrode (Rank Brothers) connected to a chart recorder. Cellular oxygen consumption was measured as described previously (50). The oxygen electrode was calibrated, assuming the concentration of oxygen in the incubation medium at $37^{\circ} \mathrm{C}$ to be $200 \mu \mathrm{M}$. Protein content in both cell and tissue samples was determined by the bicinchoninic acid protein assay.

Measurement of ATP levels. The ATP content of animal tissues was determined in $2.5 \%$ perchloric acid extracts neutralized with $\mathrm{K}_{2} \mathrm{CO}_{3}$ by reversedphase HPLC as described previously (51).

Electron microscopy. WAT, BAT, and soleus muscle were carefully removed, cut into pieces of about $1 \mathrm{~mm}^{3}$, and placed in ice-cold fixative ( $2 \%$ glutaraldehyde in $0.1 \mathrm{M}$ sodium-cacodylate buffer, $\mathrm{pH}$ 7.4) for 5 hours. Samples were then extensively washed with $0.1 \mathrm{M}$ cacodylate buffer, postfixed for 2 hours with $2 \% \mathrm{OsO}_{4}, 0.1 \mathrm{M}$ cacodylate buffer, dehydrated in ethanol, blockstained with uranyl acetate, and embedded in Epon. Ultrathin sections, collected on copper grids, were doubly stained with uranyl acetate and lead citrate and examined under a Philips CM10 transmission electron microscope. Morphometric analysis of mitochondria was performed as described previously $(9,10)$. Randomly selected areas of tissue derived from 3 animals per group were photographed at a magnification of $\times 11,500$ and analyzed with NIH Image software (http://rsb.info.nih.gov/nih-image/). Statistical analyses of cross-sectional area of mitochondria and mitochondrial densities were carried out using Prism version 2.0 software.

Statistics. Raw data from each experiment was normalized, combined, and analyzed using either an analysis of variance with Newman-Keuls multiplecomparison post hoc test or 2-tailed Student's $t$ test.

\section{Acknowledgments}

We thank Annie Higgs for her critical revision of the manuscript and Gökhan S. Hotamisligil for providing the $o b / o b, o b / o b$ p55-/-, $o b / o b$

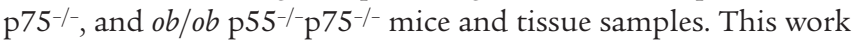
was supported by grants from the Ministero della Salute (E. Nisoli, M.O. Carruba, and E. Clementi), the Italian Association for Cancer Research (E. Clementi), the Cofinanziamento 2005 Programmi di Ricerca Scientifica di Rilevante Interesse Nazionale from the Ministero dell'Istruzione, dell'Università e della Ricerca (E. Nisoli and M.O. Carruba), and the Medical Research Council (S. Moncada).

Received for publication March 20, 2006, and accepted in revised form July 25, 2006.

Address correspondence to: Enzo Nisoli, Department of Pharmacology, Chemotherapy, and Medical Toxicology, University of Milan, via Vanvitelli, 32 - 20129 Milan, Italy. Phone: 39-0250319684; Fax: 39-02-50319682; E-mail: enzo.nisoli@unimi.it.

Alessandra Valerio and Annalisa Cardile contributed equally to this work.
1. Haslam, D.W., and James, W.P. 2005. Obesity. Lancet. 366:1197-1209.

2. Wellen, K.E., and Hotamisligil, G.S. 2005. Inflammation, stress, and diabetes. J. Clin. Invest. 115:1111-1119. doi:10.1172/JCI200525102.

3. Hotamisligil, G.S., Shargill, N.S., and Spiegelman, B.M. 1993. Adipose expression of tumor necrosis factor- $\alpha$ : direct role in obesity-linked insulin resistance. Science. 259:87-91.

4. Hotamisligil, G.S., Arner, P., Caro, J.F., Atkinson, R.L., and Spiegelman, B.M. 1995. Increased adipose tissue expression of tumor necrosis factor- $\alpha$ in human obesity and insulin resistance. J. Clin. Invest. 95:2409-2415.

5. Perreault, M., and Marette, A. 2001. Targeted disruption of inducible nitric oxide synthase protects against obesity-linked insulin resistance in muscle. Nat. Med. 7:1138-1143.

6. Wlodek, D., and Gonzales, M. 2003. Decreased energy levels can cause and sustain obesity.J. Theor. Biol. 225:33-44.

7. Boudina, S., et al. 2005. Reduced mitochondrial oxidative capacity and increased mitochondrial uncoupling impair myocardial energetics in obesity. Circulation. 112:2686-2695.

8. Munzberg, H., and Myers, M.G., Jr. 2005. Molecular and anatomical determinants of central leptin resistance. Nat. Neurosci. 8:566-570.

9. Nisoli, E., et al. 2003. Mitochondrial biogenesis in mammals: the role of endogenous nitric oxide. Science. 299:896-899.

10. Nisoli, E., et al. 2004. Mitochondrial biogenesis by $\mathrm{NO}$ yields functionally active mitochondria in mammals. Proc. Natl. Acad. Sci.U. S. A. 101:16507-16512.

11. Kelly, D.P., and Scarpulla, R.C. 2004. Transcriptional regulatory circuits controlling mitochondrial biogenesis and function. Genes Dev. 18:357-368.

12. Larrouy, D., Vidal, H., Andreelli, F., Laville, M., and Langin, D. 1999. Cloning and mRNA tissue distri- bution of human PPAR $\gamma$ coactivator-1. Int. J. Obes. Relat. Metab. Disord. 23:1327-1332.

13. Higami, Y., et al. 2004. Adipose tissue energy metabolism: altered gene expression profile of mice subjected to long-term caloric restriction. FASEBJ. 18:415-417.

14. Levin, B.E., Finnegan, M., Triscari, J., and Sullivan, A.C. 1985 . Brown adipose and metabolic features of chronic diet-induced obesity. Am. J. Physiol. 248:R717-R723.

15. Nicholls, D.G., Cunningham, S.A., and Rial, E. 1986. The bioenergetic mechanisms of brown adipose tissue thermogenesis. In Brown adipose tissue. P. Trayhurn and D.G. Nicholls, editors. Edward Arnold. London, United Kingdom. 52-85.

16. Uysal, K.T., Wiesbrock, S.M., Marino, M.W., and Hotamisligil, G.S. 1997. Protection from obesityinduced insulin resistance in mice lacking TNF- $\alpha$ function. Nature. 389:610-614.

17. Charge, S.B., and Rudnicki, M.A. 2004. Cellular and molecular regulation of muscle regeneration. Physiol. Rev. 84:209-238.

18. Jezek, P. 2002. Possible physiological roles of mitochondrial uncoupling proteins - UCPn. Int. J. Biochem. Cell Biol. 34:1190-1206.

19. Sparks, L.M., et al. 2005. A high-fat diet coordinately downregulates genes required for mitochondrial oxidative phosphorylation in skeletal muscle. Diabetes. 54:1926-1933.

20. Wisloff, U., et al. 2005. Cardiovascular risk factors emerge after artificial selection for low aerobic capacity. Science. 307:418-420.

21. Kim, J.Y., Hickner, R.C., Cortright, R.L., Dohm, G.L., and Houmard, J.A. 2000. Lipid oxidation is reduced in obese human skeletal muscle. Am. J. Physiol. Endocrinol. Metab. 279:E1039-E1044.

22. Cook, S., et al. 2003. Clustering of cardiovascular risk factors mimicking the human metabolic syndrome $\mathrm{X}$ in eNOS null mice. Swiss Med. Wkly.
133:360-363.

23. Moraes, R.C., et al. 2003. Study of the alteration of gene expression in adipose tissue of diet-induced obese mice by microarray and reverse transcriptionpolymerase chain reaction analyses. Endocrinology. 144:4773-4782.

24. Hickner, R.C., Kemeny, G., Stallings, H.W., Manning, S.M., and McIver, K.L. 2006. Relationship between body composition and skeletal muscle eNOS. Int. J. Obes. (Lond). 30:308-312.

25. Fu, W.J., et al. 2005. Dietary L-arginine supplementation reduces fat mass in Zucker diabetic fatty rats. J. Nutr. 135:714-721.

26. Xu, H., et al. 2003. Chronic inflammation in fat plays a crucial role in the development of obesity-related insulin resistance. J. Clin. Invest. 112:1821-1830. doi:10.1172/JCI200319451.

27. Weisberg, S.P., et al. 2003. Obesity is associated with macrophage accumulation in adipose tissue. J. Clin. Invest. 112:1796-1808. doi:10.1172/ JCI200319246.

28. Patti, M.E., et al. 2003. Coordinated reduction of genes of oxidative metabolism in humans with insulin resistance and diabetes: potential role of PGC1 and NRF1. Proc. Natl. Acad. Sci. U. S. A. 100:8466-8471.

29. Mootha, V.K., et al. 2003. PGC-1 $\alpha$-responsive genes involved in oxidative phosphorylation are coordinately downregulated in human diabetes. Nat. Genet. 34:267-273.

30. Wilson-Fritch, L., et al. 2004. Mitochondrial remodeling in adipose tissue associated with obesity and treatment with rosiglitazone. J. Clin. Invest. 114:1281-1289. doi:10.1172/JCI200421752.

31. Vartanian, V., et al. 2006. The metabolic syndrome resulting from a knockout of the NEIL1 DNA glycosylase. Proc. Natl. Acad. Sci. U. S. A. 103:1864-1869.

32. Lennmarken, C., Sandstedt, S., von Schenck, H., and Larsson, J. 1986. Skeletal muscle function 
and metabolism in obese women. JPEN J. Parenter. Enteral Nutr. 10:583-587.

33. Green, H.J. 1997. Mechanisms of muscle fatigue in intense exercise. J. Sports Sci. 15:247-256.

34. Bach, D., et al. 2003. Mitofusin-2 determines mitochondrial network architecture and mitochondrial metabolism. A novel regulatory mechanism altered in obesity. J. Biol. Chem. 278:17190-17197.

35. Cinti, S., et al. 2005. Adipocyte death defines macrophage localization and function in adipose tissue of obese mice and humans. J. Lipid Res. 46:2347-2355

36. Bojunga,J., Dresar-Mayert, B., Usadel, K.H., Kusterer, K., and Zeuzem, S. 2004. Antioxidative treatment reverses imbalances of nitric oxide synthase isoform expression and attenuates tissue-cGMP activation in diabetic rats. Biochem. Biophys. Res. Com mun. 316:771-780.

37. Munoz-Fernandez, M.A., and Fresno, M. 1998. The role of tumour necrosis factor, interleukin 6 , interferon-gamma and inducible nitric oxide synthase in the development and pathology of the nervous system. Prog. Neurobiol. 56:307-340.

38. Anderson, H.D., Rahmutula, D., and Gardner, D.G. 2004. Tumor necrosis factor- $\alpha$ inhibits endothelial nitric-oxide synthase gene promoter activity in bovine aortic endothelial cells. J. Biol. Chem. 279:963-969.

39. Schulze-Osthoff, K., et al. 1992. Cytotoxic activity of tumor necrosis factor is mediated by early damage of mitochondrial functions. Evidence for the involvement of mitochondrial radical generation. J. Biol. Chem. 267:5317-5323.

40. Tatsumi, T., et al. 2000. Cytokine-induced nitric oxide production inhibits mitochondrial energy production and impairs contractile function in rat cardiac myocytes. J. Am. Coll. Cardiol. 35:1338-1346.

41. Neels, J.G., Pandey, M., Hotamisligil, G.S., and Samad, F. 2006. Autoamplification of tumor necrosis factor- $\alpha$ : a potential mechanism for the maintenance of elevated tumor necrosis factor- $\alpha$ in male but not female obese mice. Am. J. Pathol. 168:435-444

42. Visser, M., Bouter, L.M., McQuillan, G.M., Wener, M.H., and Harris, T.B. 1999. Elevated C-reactive protein levels in overweight and obese adults. JAMA. 282:2131-2135.

43. Nair, S.P., Chacko, V., Arnold, C., and Diehl, A.M. 2003. Hepatic ATP reserve and efficiency of replenishing: comparison between obese and nonobese normal individuals. Am. J. Gastroenterol. 98:466-470.

44. Woods, S.C., Benoit, S.C., Clegg, D.J., and Seeley, R.J. 2004. Clinical endocrinology and metabolism. Regulation of energy homeostasis by peripheral signals. Best Pract. Res. Clin. Endocrinol. Metab. 18:497-515.

45. Selman, C., et al. 2005. Energy expenditure of calorically restricted rats is higher than predicted from their altered body composition. Mech. Ageing Dev. 126:783-793.

46. Nisoli, E., et al. 2005. Calorie restriction promotes mitochondrial biogenesis by inducing the expression of eNOS. Science. 310:314-317.

47. Kempen, K.P., Saris, W.H., Kuipers, H., Glatz, J.F., and Van Der Vusse, G.J. 1998. Skeletal muscle metabolic characteristics before and after energy restriction in human obesity: fibre type, enzymatic beta-oxidative capacity and fatty acid-binding protein content. Eur. J. Clin. Invest. 28:1030-1037.

48. Rodbell, M. 1964. Metabolism of isolated fat cells. I. Effects of hormones on glucose metabolism and lipolysis. J. Biol. Chem. 239:375-380.

49. Cossu, G., et al. 1980. In vitro differentiation of satellite cells isolated from normal and dystrophic mammalian muscles. A comparison with embryonic myogenic cells. Cell Differ. 9:357-368.

50. Clementi, E., Brown, G.C., Feelisch, M., and Moncada, S. 1998. Persistent inhibition of cell respiration by nitric oxide: crucial role of S-nitrosylation of mitochondrial complex I and protective action of glutathione. Proc. Natl. Acad. Sci. U. S. A. 95:7631-7636.

51. Stocchi, V., et al. 1985. Simultaneous extraction and reverse-phase high-performance liquid chromatographic determination of adenine and pyridine nucleotides in human red blood cells. Anal. Biochem. 146:118-124. 